\title{
Potential of circulating tumor cells as blood-based biomarkers in cancer liquid biopsy
}

\begin{abstract}
"In the future, circulating tumor cells may dramatically improve our ability to monitor malignancies and indicate whether or not a treatment may work, arguing for complementary roles of conventional and liquid biopsies."
\end{abstract}

First draft submitted: 28 October 2015; Accepted for publication: 28 October 2015; Published online: 22 January 2016

Keywords: blood-based biomarkers $\bullet$ circulating tumor cells $\bullet$ liquid biopsy $\bullet$ personalized medicine $\bullet$ tumor heterogeneity

Despite the first reports of circulating cells from solid tumors shed light on the circulation in deceased and in living patients in 1869 [1] and the mid 1950s [2], respectively, it took almost 50 years until the introduction of the CellSearch ${ }^{\circledR}$ system by Veridex (now Janssen Diagnostics, LLC, Raritan, NJ, USA) as well as its approval by the US FDA [3] in 2004 paved the way for widespread clinical assessment of the number of circulating tumor cells (CTCs) as a prognostic diagnostic tool. The technology is based on enumeration of cells expressing the epithelial marker EpCAM and excludes cells positive for the hematopoietic cell surface marker CD45. The number of EpCAM+CD45cells correlate with prognosis and are being used to monitor the response to chemotherapy in metastatic breast [4], prostate [5] and colorectal cancer [6]. This information can be used to spare cancer patients who are not responding to therapy the burden of adverse effects $[7,8]$. Moreover, CTCs harbor the potential to provide tumor-specific molecular information that can guide chemotherapy and targeted therapies in a personalized medicine approach. In recent years, a number of new technologies have been introduced that exploit biological and physical properties of CTCs apart from EpCAM as a surface marker. The dependence of CTC isolation on EpCAM expression may lead to an underestimation of the actual number of CTCs by missing tumor cells that underwent epithelial-to-mesenchymal transition (EMT). EMT is considered to be an event associated with metastasis and adoption of a stem cell phenotype by tumor cells $[9,10]$. It remains to be elucidated though, whether and how the number and phenotype of EpCAM negative/EMT marker positive CTCs correlate to disease progression [11].

Currently, predictive biomarkers are codeveloped as companion diagnostics in almost $50 \%$ of the cases with newly approved targeted cancer therapeutics [12]. The trivial explanation for this need lies in the ineffectiveness of targeted therapy when the therapeutic target is not expressed or is not the driver of tumor growth in the presence of other oncogenic molecular alterations in the tumor. A lack of suitable predictive biomarkers therefore causes attrition in clinical development, unnecessary treatment burden and cost for the patients and payers, respectively. With the introduction of an ever increasing number of targeted therapies in all tumor indications, the percentage of companion diagnostics guiding the use of new targeted therapeutics is expected to rise further. This process is driven by the pharmaceutical industry with the motivation to decrease

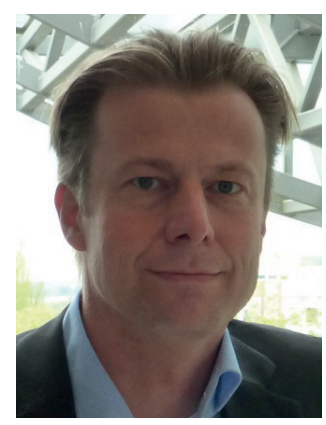

Thomas Schlange Bayer Pharma AG, Global Biomarker Research, 42096 Wuppertal, Germany

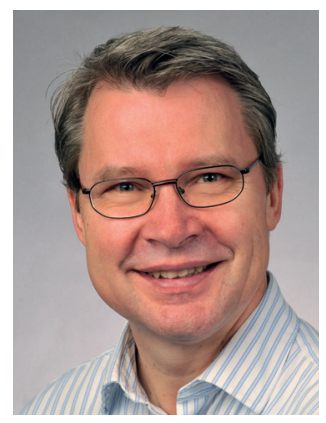

Klaus Pantel

Author for correspondence: Department of Tumour Biology, University Medical Center HamburgEppendorf, 20246 Hamburg, Germany pantel@uke.de 
cost, time and risk of cancer drug development programs, but also by regulators, payers and patients in an effort to increase safety, efficacy and cost-to-benefit ratio of new drugs.

Most solid tumor diagnostics approaches are dependent on the availability of tumor biopsies and either immunohistochemical detection of protein expression or the PCR- or sequencing-based analysis of mRNA expression of a therapeutic target [13]. There are, however, disadvantages of solid tumor biopsies. In the case of minimal residual disease either after successful surgery, chemo or radiation therapy, there is obviously no material that could be probed. Nevertheless, micrometastases may be present and cause relapse that may be detected too late for effective treatment. In several tumor indications or when metastases are not resectable due to their location or number, biopsies are difficult to obtain or associated with significant risk for the patient [14]. This is the case, for example, in lung cancer where bleeding may cause complications during the process of taking a biopsy. In addition, the relative inaccessibility of biopsy material is often prohibitive when longitudinal sampling is required in order to monitor disease progression or development of acquired resistance under therapy. A major obstacle for the interpretation of biopsy material is intratumoral heterogeneity that may result in poor representation (e.g., in stromarich tumors like pancreatic carcinoma) or even failure to detect the expression of relevant markers (e.g., scattered expression of proteins like c-MET) [15]. Rare or emerging molecular phenotypes responsible for the development of treatment resistance, relapse or metastasis may not be recognized. In addition, the phenotype of metastases can differ from the primary tumor and in-between metastases at different locations [16]. The biopsy of the latter might therefore not be informative for the choice of treatments effective against metastatic disease [17].

\section{"An important point that needs more attention is the fact that the interpretation of genetic information may not be sufficient to elucidate the activated pathways that are ultimately the target of therapeutic intervention."}

The concept of liquid biopsy based on biomarkers such as CTCs and circulating free tumor DNA is considered to be providing information that can mitigate the shortcomings of conventional tumor biopsies. The CTC segment of this market alone is expected to reach a value of $\$ 2.1$ to $\$ 4.3$ billion by 2020 [18]. These calculations are largely based on the currently marketed technologies based on EpCAM expression and microfluidics with breast, colorectal and prostate cancer as dominant indications. By adding further indications to this list and demonstrating more widespread value of liquid biopsy in patient stratification, prediction of treatment response and treatment monitoring, sustained growth appears feasible.

Today, counting CTCs already provides information that is able to support therapeutic decisions. The monitoring of the number of CTCs serves as a pharmacodynamic marker where treatment reduces or eliminates CTCs and this has an impact on the prognosis of the patient $[5,7,8,19,20]$. Yet, by focusing on enumeration of CTCs as the current diagnostic standard only, molecular information that is crucial to inform clinicians and patients about treatment options is lacking. Many groups have, therefore, focused on the biochemical and molecular characterization of CTCs and encouraging first results indicate that this approach might provide important information for the management of cancer patients, for example, androgen receptor variant-7 expression in CTCs predicts resistance to antiandrogen therapy in prostate cancer [21]. The challenge for future applications of CTCs as diagnostic tools will clearly be to allow capturing of a sufficient number of CTCs in a given patient that represents the heterogeneity of the metastatic burden. Which CTC count is sufficient to provide this information depends on the molecular target and can be only tested in clinical trials with defined end points such as progression-free or overall survival. Only with resolution at the single cell level, disease heterogeneity can be reflected and a true unique value of liquid biopsies be leveraged. Advances in analytical technologies such as whole genome amplification [22,23], digital PCR $[24,25]$ and next-generation sequencing [26] already provide the possibility to perform such analyses. However, like for CTC capturing apart from the CellSearch system, a standardization of such technologies down to the preanalytical stage has not been achieved. In order to meet this need for standard operating procedures, the international CANCER-ID consortium within the framework of the EU's innovative medicines initiative has been formed in 2014 [27]. Until 2019, partners from academic and clinical research, small-to-medium sized enterprises and the pharmaceutical industry will collaborate to develop such standards for technologies in the liquid biopsies field.

Approaches relying on additional markers for CTC isolation (e.g., EMT markers or markers with a certain indication specificity such as HER2) have been introduced [28,29]. Biophysical properties such as cell size and rigidity are being exploited by filtration and microfluidic devices to improve the yield [30] and the latter offering the additional advantage that living CTCs can be isolated, enabling cultivation methods that may help interrogating the phenotype and response to 
therapeutics [31-33]. The development of a strategy for downstream analysis is a crucial issue as it determines preanalytical sample handling, single cell identification and isolation as well as processing of isolated cells for DNA, RNA and protein analysis.

Once single cells are isolated, their origin from the primary tumor or metastases needs to be confirmed. To achieve this goal, genetic alterations or specific tumor markers previously identified in biopsy material can serve as proof. Immunohistochemical and FISH methods [34] have been combined to this end. As with all optical methods for CTC identification and data documentation, the use of such image-based analysis requires the standardization of image acquisition and analysis. This exercise has been extensively performed with the CellSearch system [35] and will have to be done with emerging technologies that are based on image analysis to achieve regulatory approval as companion diagnostic. Undoubtedly, such companion diagnostics would offer great potential in disease monitoring, for example, by following the expression of therapeutic targets such as the immune checkpoint modulator programmed death-ligand $1[36,37]$ or stemness markers that might be correlated with responsiveness to chemotherapy $[38,39]$ in CTCs.

Genetic analyses allow the identification of driver mutations and mutations that may lead to resistance toward targeted therapies [19,40-42]. An important point that needs more attention is the fact that the interpretation of genetic information may not be sufficient to elucidate the activated pathways that are ultimately the target of therapeutic intervention. This requires the protein- or phosphor-protein-based analysis of CTCs, again ideally down to the single cell level [43]. The possibility to perform such analysis would also add an advantage over the sole analysis of circulating tumor DNA.

\section{References}

1 Ashworth TR. A case of cancer in which cells similar to those in the tumor were seen in the blood after death. Med. J. Aust. 1, 146-147 (1869).

2 Engell HC. Cancer cells in the circulating blood; a clinical study on the occurrence of cancer cells in the peripheral blood and in venous blood draining the tumour area at operation. Acta Chir. Scand. Suppl. 201, 1-70 (1955).

3 FDA. Re: K031588 evaluation of automatic class III designation. Cell search epithelial cell kit/cell spotter analyzer.

www.accessdata.fda.gov

4 Hayes DF, Cristofanilli M, Budd GT et al. Circulating tumor cells at each follow-up time point during therapy of metastatic breast cancer patients predict progression-free and overall survival. Clin. Cancer. Res. 12 (14 Pt 1), 4218-4224 (2006).
In summary, the current knowledge on CTCs gives rise to the hope that information from these cells reflects the heterogeneity and dynamic nature of the disease in a longitudinal fashion without increasing the burden on the patient that is inflicted by multiple surgical biopsies. Further progress in the field depends on a deeper understanding of the significance of CTC subpopulations in different tumor indications, in early stage and minimal residual disease. Molecular analysis of sufficient numbers of single CTCs might aid the development of predictive markers for treatment efficacy and emerging resistance. Given that the turnaround times of CTC-based analyses are short enough, there is great potential that therapy decisions can be guided by these technologies. The genetic and signaling pathway activation data collected by analyzing solid tumor biopsies and during surgical resection of primary tumor and metastases do already provide valuable insight into a patient's disease and therapy options. In the future, CTCs may dramatically improve our ability to monitor malignancies and indicate whether or not a treatment may work, arguing for complementary roles of conventional and liquid biopsies.

\section{Financial \& competing interests disclosure}

T Schlange is full-time employee and share holder of Bayer Pharma AG, Germany. This work has received funding from the European Union's Seventh Framework Programme (FP7/2007-2013) for the Innovative Medicine Initiative under grant agreement no (115749). The authors have no other relevant affiliations or financial involvement with any organization or entity with a financial interest in or financial conflict with the subject matter or materials discussed in the manuscript apart from those disclosed.

No writing assistance was utilized in the production of this manuscript.

5 De Bono JS, Scher HI, Montgomery RB et al. Circulating tumor cells predict survival benefit from treatment in metastatic castration-resistant prostate cancer. Clin. Cancer. Res. 14(19), 6302-6309 (2008).

6 Cohen SJ, Punt CJ, Iannotti N et al. Relationship of circulating tumor cells to tumor response, progression-free survival, and overall survival in patients with metastatic colorectal cancer. J. Clin. Oncol. 26(19), 3213-3221 (2008).

7 Bidard FC, Peeters DJ, Fehm T et al. Clinical validity of circulating tumour cells in patients with metastatic breast cancer: a pooled analysis of individual patient data. Lancet Oncol. 15(4), 406-414 (2014).

8 Scher HI, Heller G, Molina A et al. Circulating tumor cell biomarker panel as an individual-level surrogate for survival in metastatic castration-resistant prostate cancer. J. Clin. Oncol. 33(12), 1348-1355 (2015).

9 Brabletz T. To differentiate or not - routes towards metastasis. Nat. Rev. Cancer 12(6), 425-436 (2012). 
10 Ye X, Tam WL, Shibue T et al. Distinct EMT programs control normal mammary stem cells and tumour-initiating cells. Nature 525(7568), 256-260 (2015).

11 Alix-Panabieres C, Pantel K. Challenges in circulating tumour cell research. Nat. Rev. Cancer 14(9), 623-631 (2014).

12 Circulating tumor cells (CTCs) and cancer stem cells (CSCs) 2013 market report. Research trends and clinical utility. 134 (2013).

13 Wan L, Pantel K, Kang Y. Tumor metastasis: moving new biological insights into the clinic. Nat. Med. 19(11), 1450-1464 (2013)

14 Alix-Panabieres C, Pantel K. Circulating tumor cells: liquid biopsy of cancer. Clin. Chem. 59(1), 110-118 (2013).

15 Chen Z, Fillmore CM, Hammerman PS, Kim CF, Wong KK. Non-small-cell lung cancers: a heterogeneous set of diseases. Nat. Rev. Cancer 14(8), 535-546 (2014).

16 Gerlinger M, Rowan AJ, Horswell S et al. Intratumor heterogeneity and branched evolution revealed by multiregion sequencing. N. Engl. J. Med. 366(10), 883-892 (2012).

17 Niikura N, Liu J, Hayashi N et al. Loss of human epidermal growth factor receptor 2 (HER2) expression in metastatic sites of HER 2 -overexpressing primary breast tumors. J. Clin. Oncol. 30(6), 593-599 (2012).

18 Rusu A. The market for circulating tumor cells (CTCs) and cancer stem cells (CSCs). Kalorama Information Market Intelligence Report (2015).

www.rnrmarketresearch.com

19 Punnoose EA, Atwal S, Liu Wet al. Evaluation of circulating tumor cells and circulating tumor DNA in non-small cell lung cancer: association with clinical endpoints in a Phase II clinical trial of pertuzumab and erlotinib. Clin. Cancer Res. 18(8), 2391-2401 (2012).

20 Dawood S, Cristofanilli M. Using circulating tumor cells to guide therapy in breast cancer: could this replace biopsies? Pharmacogenomics 16(7), 669-672 (2015).

21 Antonarakis ES, Lu C, Wang $\mathrm{H}$ et al. AR-V7 and resistance to enzalutamide and abiraterone in prostate cancer. N. Engl. J. Med. 371(11), 1028-1038 (2014).

22 Gasch C, Pantel K, Riethdorf S. Whole genome amplification in genomic analysis of single circulating tumor cells. Methods Mol. Biol. 1347 221-232 (2015).

23 Heitzer E, Auer M, Gasch C et al. Complex tumor genomes inferred from single circulating tumor cells by array-CGH and next-generation sequencing. Cancer Res. 73(10), 2965-2975 (2013).

24 Guttery DS, Page K, Hills A et al. Noninvasive detection of activating estrogen receptor 1 (ESR1) mutations in estrogen receptor-positive metastatic breast cancer. Clin. Chem. 61(7), 974-982 (2015).

25 Olsson E, Winter C, George A et al. Serial monitoring of circulating tumor DNA in patients with primary breast cancer for detection of occult metastatic disease. EMBO Mol. Med. 7(8), 1034-1047 (2015).

26 Kato S, Janku F. Cell-free DNA as a novel marker in cancer therapy. Biomark. Med. 9(7), 703-712 (2015).
27 Cancer Id. www.cancer-id.eu

28 Agelaki S, Kalykaki A, Markomanolaki H et al. Efficacy of lapatinib in therapy-resistant HER2-positive circulating tumor cells in metastatic breast cancer. PLoS ONE 10(6), e0123683 (2015).

29 Riethdorf S, Muller V, Zhang L et al. Detection and HER2 expression of circulating tumor cells: prospective monitoring in breast cancer patients treated in the neoadjuvant GeparQuattro trial. Clin. Cancer Res. 16(9), 2634-2645 (2010).

30 Joosse SA, Gorges TM, Pantel K. Biology, detection, and clinical implications of circulating tumor cells. EMBO Mol. Med. 7(1), 1-11 (2015).

31 Yu M, Bardia A, Aceto N et al. Cancer therapy. Ex vivo culture of circulating breast tumor cells for individualized testing of drug susceptibility. Science 345(6193), 216-220 (2014).

32 Khoo BL, Lee SC, Kumar P et al. Short-term expansion of breast circulating cancer cells predicts response to anti-cancer therapy. Oncotarget 6(17), 15578-15593 (2015).

33 Cayrefourcq L, Mazard T, Joosse S et al. Establishment and characterization of a cell line from human circulating colon cancer cells. Cancer Res. 75(5), 892-901 (2015).

34 Pailler E, Adam J, Barthelemy A et al. Detection of circulating tumor cells harboring a unique ALK rearrangement in ALK-positive non-small-cell lung cancer. J. Clin. Oncol. 31(18), 2273-2281 (2013).

35 Coumans F, Terstappen L. Detection and characterization of circulating tumor cells by the CellSearch approach. Methods Mol. Biol. 1347, 263-278 (2015).

36 Soria JC, Marabelle A, Brahmer JR, Gettinger S. Immune checkpoint modulation for non-small cell lung cancer. Clin. Cancer Res. 21(10), 2256-2262 (2015).

37 Mazel M, Jacot W, Pantel K et al. Frequent expression of PD-L1 on circulating breast cancer cells. Mol. Oncol. 9(9), 1773-1782 (2015).

38 Valladares-Ayerbes M, Blanco-Calvo M, Reboredo $\mathrm{M}$ et al. Evaluation of the adenocarcinoma-associated gene AGR2 and the intestinal stem cell marker LGR 5 as biomarkers in colorectal cancer. Int. J. Mol. Sci. 13(4), 4367-4387 (2012).

39 Kobayashi S, Yamada-Okabe H, Suzuki M et al. LGR5positive colon cancer stem cells interconvert with drugresistant LGR5-negative cells and are capable of tumor reconstitution. Stem Cells 30(12), 2631-2644 (2012).

40 Maheswaran S, Sequist LV, Nagrath S et al. Detection of mutations in EGFR in circulating lung-cancer cells. N. Engl. J. Med. 359(4), 366-377 (2008).

41 Crowley E, Di Nicolantonio F, Loupakis F, Bardelli A. Liquid biopsy: monitoring cancer-genetics in the blood. Nat. Rev. Clin. Oncol. 10(8), 472-484 (2013).

42 Krebs MG, Metcalf RL, Carter L, Brady G, Blackhall FH, Dive C. Molecular analysis of circulating tumour cells-biology and biomarkers. Nat. Rev. Clin. Oncol. 11(3), 129-144 (2014).

43 Kim P, Liu X, Lee T et al. Highly sensitive proximity mediated immunoassay reveals HER2 status conversion in the circulating tumor cells of metastatic breast cancer patients. Proteome Sci 9(1), 75 (2011). 